\title{
Estudos sobre análise de assunto no Brasil: estado da arte e perspectivas futuras
}

Estudios sobre el análisis de contenido en Brasil: estado de la cuestión y perspectivas futuras

Studies about subject analysis in Brazil: research and future perspectives

\section{Paula Regina Dal'Evedove (1), Roberta Cristina Dal'Evedove TARTAROtti (2) E Mariângela Spotti Lopes FUJITA (3)}

\begin{abstract}
(1) Universidade Federal de São Carlos, Av. Washington Luís, s/n, CEP. 13565-905, São Carlos, SP, Brasil, dalevedove@ufscar.br, (2 e 3) Universidade Estadual Paulista, Av. Hygino Muzzi Filho, 737, Campus Universitário, CEP.17525900, Marília, SP, Brasil, roberta_tartarotti@yahoo.com.br, mariangelaslf57@gmail.com
\end{abstract}

\begin{abstract}
Resumen
Este estudio pretende trazar un mapa y discutir el conocimiento producido sobre análisis de contenido en la Ciencia de la Información brasileña. Con este objetivo, se llevó a cabo una pesquisa bibliográfica de los artículos científicos publicados en revistas brasileñas en el ámbito de la Ciencia de la Información, utilizando como términos de búsqueda "análisis de contenido" y "análisis conceptual". El análisis del corpus de investigación permitió, entre otros resultados, la identificación de las referencias teóricas actuantes en este universo y las tendencias actuales en la literatura nacional de Ciencia de la Información, lo que permitió esbozar una visión general de los principales desafíos y perspectivas de futuro para la pesquisa y desarrollo en análisis de contenido.
\end{abstract}

Palabras clave: Análisis de contenido. Investigación. Estado de la cuestión. Brasil.

\section{Introdução}

O desenvolvimento científico e consequente crescimento da produção científica nas últimas décadas levaram à necessidade de criação de instrumentos que possibilitassem tanto a gestão como uma melhor recuperação dos documentos em sistemas de informação, especialmente por assunto, o que nos leva a duas dimensões: a representação e a recuperação temática da informação. Esta atividade, denominada indexação, é percebida como um processo intelectual realizado pelo indexador, que tem por base a compreensão do texto e a representação dos documentos. Como abordagem teórica da área de Organização e Representação do Conhecimento, a indexação viabiliza a representação temática dos documentos, com fins de recuperação por assunto em sistemas de recuperação da informação.

Neves, Dias e Pinheiro (2006) esclarecem que as pesquisas sobre indexação têm evidenciado três pontos: compreensão de texto (identificação do conteúdo de um documento); produção de texto

\begin{abstract}
The present study aims to map and discuss the knowledge produced on the subject analysis in Brazilian Information Science. For that, a bibliographic research was carried out by means of the survey of scientific articles published in national journals of the area of Information Science, using search terms: "subject analysis" and "conceptual analysis". The analysis of the research corpus provided, among others, the identification of the theoretical referents in this universe and the current trends in the national literature of Information Science, which allowed to outline a panorama of the main challenges and future perspectives of research and development in subject analysis.
\end{abstract}

Keywords: Subject analysis. Research. State of the art. Brazil.

(tradução do conteúdo identificado para a linguagem de indexação e/ou sua expressão na forma de um resumo); e a representação do conteúdo (criação de vocabulários controlados, como os tesauros).

Conceitualmente, a indexação de assuntos é um processo formado por subprocessos ou etapas que tem como objetivos identificar o conteúdo de um documento e representá-lo por meio de uma metalinguagem construída, com o intuito de promover a efetiva recuperação da informação (Tartarotti e Boccato, 2013). Nesta concepção, entendemos o processo de indexação mediante determinação do assunto do documento e tradução do assunto por termos representáveis em linguagens de indexação, ou seja, representação por conceitos e representação por linguagens construídas (sistemas de classificação, cabeçalhos de assunto ou tesauros). A divisão em duas etapas não é consensual entre teóricos e pesquisadores da área dedicados à questão, podendo-se encontrar indicações de três, quatro, cinco ou mais etapas compreendidas pelo processo (Chan, 1994; Cleveland e Cleveland, 1990; ISO 
5963, 1985; Langridge, 1989; Chu e O'Brien, 1993; Mai, 1997; 2000; Van Slype, 1991; Tamayo e Valdez, 2008; dentre outros).

Apesar das diferenças em termos teóricos e práticas adotadas, Fujita, Rubi e Boccato (2009, p. 24) explicam que as etapas do processo de indexação não precisam ser necessariamente realizadas de maneira sequencial, visto que o indexador profissional, já familiarizado com o processo, pode realizá-las simultaneamente e até de maneira automática. Neste sentido, quanto mais familiarizado com a área científica especializada no qual atua, mais estas etapas são superpostas durante o processo de indexação pelo profissional. De acordo com Mai (2000), a ação tomada por indexadores com diferentes níveis de experiência pode não ser exatamente a mesma, mas as etapas e elementos do processo de indexação de assunto são considerados fundamentais para qualquer processo de indexação.

Como etapa inicial, a análise de assunto, também denominada por análise temática ou análise conceitual, é considerada na literatura e na prática profissional como sendo a mais importante do processo de indexação, pois determina os resultados das estratégias de busca realizada pelo usuário no momento da recuperação da informação. Sendo, portanto, a "operação base para todo o procedimento de recuperação de informações" (Cesarino e Pinto, 1980, p. 32). Esta etapa se efetiva mediante a leitura documental, momento em que os conceitos tratados em um documento são identificados e selecionados para posterior representação em termos de indexação. Em outras palavras, a análise de assunto decidirá sobre os principais tópicos do assunto de um documento, precedendo a fase de tradução desses tópicos de acordo com a linguagem de indexação adotada pelo sistema de recuperação de informação (Chu e O'brien, 1993).

Considerando-se que a construção de qualquer texto é baseada em um ou mais temas, é por meio da leitura profissional na representação temática da informação que ocorre a busca pela tematicidade documental, revelada na análise de assunto. Assim, a análise de assunto é realizada durante a leitura documental, sendo, por sua vez, subdividida em outros três estágios: compreensão do conteúdo do documento; identificação dos conceitos que representam este conteúdo; e seleção dos conceitos válidos para recuperação. É a leitura do documento que dá início à atividade de indexação, diferindo de uma leitura normal ao exigir procedimentos diferentes, "ainda que os conhecimentos necessários para um bom entendimento de um texto sejam comuns a ambas" (Fujita, 2003, p. 84).
Durante a análise de assunto, o indexador examina o documento procurando compreender o texto por meio da identificação do assunto, com o objetivo posterior de selecionar os conceitos indexáveis. A utilização e o domínio de normas e metodologias de análise pelo indexador possibilitam bons resultados na recuperação, o que pressupõe o conhecimento de abordagens sistematizadas ao texto, cuja eficácia desta atividade pode ser avaliada frente aos resultados obtidos pelo usuário no momento da recuperação da informação (Fujita, 2003). Sendo assim, a análise de assunto implica em determinar a tematicidade (tema, tópico ou atinência) do documento mediante a identificação e seleção dos conceitos que comporão o assunto principal e secundários. Constitui-se, portanto, em uma etapa complexa e subjetiva que exige todo um trabalho intelectual por parte do indexador na construção de sentidos e significados.

Sendo notória a sua importância no processo de indexação, a proposta neste estudo foi avaliar o estado da arte das pesquisas brasileiras sobre análise de assunto. Para tanto, apresenta a trajetória das pesquisas publicadas em formato de artigo científico para compreender a atual constituição e enfoque da temática, de modo a contribuir com o avanço das produções intelectuais dos pesquisadores da Ciência da Informação brasileira.

\section{Procedimentos metodológicos}

De modo a mapear as pesquisas sobre análise de assunto conduzidas pela comunidade brasileira de Ciência da Informação, o estudo desenvolveu-se como pesquisa exploratória e descritiva, com uso de fontes bibliográficas para aproximação, abordagem e tratamento do objeto de estudo proposto. Portanto, recorreu-se às pesquisas anteriores para verificar a produção de conhecimento em análise de assunto no cenário nacional.

Para uma melhor aproximação com o objeto de estudo, a pesquisa foi pautada em uma sequência ordenada de procedimentos relativos à pesquisa bibliográfica apresentados por Salvador (1986), delimitados em: a) escolha do assunto e formulação do problema de pesquisa; b) investigação das soluções a partir da coleta da documentação; c) análise explicativa das soluções; e d) síntese integradora (análise, reflexão e proposição de soluções mediante leitura, anotações e indagações do material).

Delimitou-se como corpus de análise pesquisas publicadas em formato de artigo científico em periódicos brasileiros de Ciência da Informação em razão do importante papel que estes canais de 
comunicação formal desempenham para a divulgação e promoção das pesquisas acadêmicocientíficas realizadas na área.

Foram coletados todos os artigos que continham os termos "análise de assunto", "análise de conteúdo", "análise temática" e "análise conceitual" mediante busca nos campos de pesquisa dos periódicos científicos pertencentes aos estratos $\mathrm{A} 1$ até B2, conforme lista de classificação de periódicos Qualis 2017 elaborada pela Coordenação de Aperfeiçoamento de Pessoal de Nível Superior (CAPES). A busca realizada em março de 2017 na Base de Dados Referencial de Artigos de Periódicos em Ciência da Informação (BRAPCl) viabilizou a localização de 37 artigos que foram analisados por meio da leitura do título, resumo e palavras-chave, com o propósito de verificar a pertinência dos mesmos à temática em estudo. Sendo desconsiderados os trabalhos que faziam alusão de forma muito indireta ao tema, o corpus de pesquisa foi constituído por 31 artigos que datam de 1980 a 2016. Para a elaboração das redes, utilizou-se o software Ucinet.

\section{Resultados e discussão}

No primeiro momento, empregou-se a estatística descritiva para a apresentação dos dados referentes às características gerais do corpus da pesquisa. Os 31 artigos recuperados estão distribuídos em 15 diferentes periódicos. O periódico com maior destaque é Perspectivas em Ciência da Informação, concentrando $16 \%$ do total de artigos publicados na temática análise de assunto. Ciência da Informação, DataGramaZero e Informação \& Sociedade concentram $10 \%$ dos estudos cada. Juntos, os periódicos mencionados representam $46 \%$ da produção brasileira na temática. Outro grupo formado pelos periódicos Brazilian Journal of Information Science, Informação \& Informação, PontodeAcesso, Revista de Biblioteconomia de Brasília, Revista Digital de Biblioteconomia e Ciência da Informação e Tendências da Pesquisa Brasileira em Ciência da Informação concentra $39 \%$ da produção total ou $6,5 \%$ cada; seguido do grupo constituído pelos periódicos $\mathrm{Bi}$ blionline, Em Questão, Encontros Bibli, Revista ACB e Transinformação com apenas $3 \%$ dos artigos cada, conforme segue (Tabela I).

Dos 33 autores que publicaram na temática, destaca-se Fujita com 11 artigos, seguida de Dias, Naves, Neves e Redigolo, ambos com 3. Um terceiro grupo de autores composto por Cervantes, Lima, Maculan, Rodrigues e Rubi publicaram 2 artigos cada. Já com apenas 1 artigo aparecem os autores Almeida, Alves, Boccato, Carvalho, Cavalcanti, Cesarino, Cordeiro, Dal'Evedove, Duarte, Ferreira, Gonçalves, Marinho, Moraes,
Moura, Nascimento, Novellino, Oliveira, Pinheiro, Pinho, Pinto, Silva, Sousa e Souza.

\begin{tabular}{ll}
\hline Periódicos & $N$. \\
\hline Perspectivas em Ciência da Informação & 5 \\
\hline Ciência da Informação & 3 \\
\hline DataGramaZero & 3 \\
\hline Informação \& Sociedade & 3 \\
\hline Brazilian Journal of Information Science & 2 \\
\hline Informação \& Informação & 2 \\
\hline PontodeAcesso & 2 \\
\hline Revista de Biblioteconomia de Brasília & 2 \\
\hline $\begin{array}{l}\text { Revista Digital de Biblioteconomia e Ciência da } \\
\text { Informação }\end{array}$ & 2 \\
\hline $\begin{array}{l}\text { Tendências da Pesquisa Brasileira em Ciência da } \\
\text { Informação }\end{array}$ & 2 \\
\hline Biblionline & 1 \\
\hline Em Questão & 1 \\
\hline Encontros Bibli & 1 \\
\hline Revista ACB & 1 \\
\hline
\end{tabular}

Tabela I. Distribuição dos artigos nos periódicos de Ciência da Informação

\begin{tabular}{ll}
\hline Autores & N. \\
\hline Fujita, M. S. L. & 11 \\
\hline Dias, E. W. & 3 \\
\hline Naves, M. M. L. & 3 \\
\hline Neves, D. A. de B. & 3 \\
\hline Redigolo, F. M. & 3 \\
\hline Cervantes, B. M. N. & 2 \\
\hline Lima, G. A. B. de O. & 2 \\
\hline Maculan, B. C. M. dos S. & 2 \\
\hline Rodrigues, M. R. & 2 \\
\hline Rubi, M. P. & 2 \\
\hline Almeida, C. C. de & 1 \\
\hline Alves, R. C. V. & 1 \\
\hline Boccato, V. R. C. & 1 \\
\hline Carvalho, M. A. & 1 \\
\hline Cavalcanti, H. C. & 1 \\
\hline Cesarino, M. A. da N. & 1 \\
\hline
\end{tabular}
de assunto no Brasil: estado da arte e perspectivas futuras. // Scire. 24:1 (en.-jun. 2018) 35-44. ISSN 1135-3716. 


\begin{tabular}{ll}
\hline Cordeiro, R. I. de N. & 1 \\
\hline Dal'Evedove, P. R. & 1 \\
\hline Duarte, F. A. & 1 \\
\hline Ferreira, A. C. & 1 \\
\hline Gonçalves, E. F. & 1 \\
\hline Marinho, A. C. M. & 1 \\
\hline Moraes, J. B. E. de & 1 \\
\hline Moura, M. A. & 1 \\
\hline Nascimento, F. A. & 1 \\
\hline Novellino, M. S. F. & 1 \\
\hline Oliveira, R. A. de & 1 \\
\hline Pinheiro, A. M. V. & 1 \\
\hline Pinho, F. A. & 1 \\
\hline Pinto, M. C. M. F. & 1 \\
\hline Silva, M. dos R. da & 1 \\
\hline Sousa, B. P. de & 1 \\
\hline Souza, R. F. & 1 \\
\hline
\end{tabular}

Tabela II. Autores mais produtivos



Figura 1. Representação gráfica da rede de coautoria

Ao elaborarmos a rede de coautoria, temos a seguinte representação gráfica (Figura 1). Conforme apresentado, percebe-se uma rede não muito densa, sendo que a maior subrede, encabeçada por Fujita é composta pelos seguintes autores: Rubi, Boccato, Silva, Sousa, Dal'Evedove, Redigolo, Moraes, Almeida, Alves, Neves, Oliveira e Gonçalves. Outras subredes são compostas, primeiramente, pelos autores Pinho com Marinho e Nascimento; e Dias, Naves e Moura. Os autores Ferreira, Maculan e Lima formam outra subrede. Três subredes aparecem com apenas dois autores, compostas por Cesarino com Pinto; Rodrigues com Cervantes; e Cavalcanti com Carvalho. Dentre os autores que não realizaram colaboração com os demais, aparecem Cordeiro, Novellino, Souza e Duarte. De forma geral, existe uma forte tendência na concentração de colaboração científica entre autores da mesma universidade, indicando subgrupos de pesquisa isolados.

Em relação às temáticas presentes nos artigos, foram identificadas 110 palavras-chave, sendo que um artigo de 1980 não menciona e em um dos artigos as palavras-chave estavam em inglês, tendo sido traduzidas. Ao agruparmos as que se repetiram, no total foram identificadas 100 diferentes palavras-chave nos artigos relacionados à temática análise de assunto.

\begin{tabular}{lc}
\hline Palavras-chave & $N$. \\
\hline Análise de assunto & 14 \\
\hline Indexação & 13 \\
\hline Leitura documental & 4 \\
\hline Bibliotecas universitárias & 3 \\
\hline Catalogação de assunto & 3 \\
\hline Indexador & 3 \\
\hline Tratamento temático da informação & 3 \\
\hline Análise documental & 2 \\
\hline Identificação de conceitos & 2 \\
\hline Mapas conceituais & 2 \\
\hline Modelo de leitura técnica & 2 \\
\hline Organização da informação & 2 \\
\hline Organização e representação do conhecimento & 2 \\
\hline Representação da informação & 2 \\
\hline
\end{tabular}

Tabela III. Palavras-chave identificadas nos artigos

De acordo com os dados apresentados, as palavras-chave que mais apareceram foram primeiramente Análise de assunto, com 14 ocorrências, seguida por Indexação com 13 ocorrências, como esperado. Na sequência, aparecem Leitura documental, Bibliotecas universitárias, Catalogação de assunto, Indexador e Tratamento temáde assunto no Brasil: estado da arte e perspectivas futuras. // Scire. 24:1 (en.-jun. 2018) 35-44. ISSN 1135-3716. 
tico da informação com 3 ocorrências cada, demonstrando a tendência de pesquisas sobre análise de assunto em bibliotecas universitárias. Com 2 ocorrências estão Análise documental, Identificação de conceitos, Mapas conceituais, Modelo de leitura técnica, Organização da informação, Organização e representação do conhecimento e Representação da informação. As demais palavras-chave apareceram apenas 1 vez nos artigos, não tendo relevância significativa: Abordagem sociocognitiva; Abordagem sociocognitiva qualitativa; Análise conceitual; Áreas de ciência e tecnologia; ARIST; Artigo científico; Atinência; Atuação profissional; Catálogos coletivos online; Ciclo junino; Ciências cognitivas; Classificação do conhecimento; Contexto profissional; Ensino de indexação; Estudo bibliométrico; Festas populares; Fontes de informação visual; História da indexação; Imagens e filmes; Indexação - NBR 12676/1992; Indexação - teoria; Indexação de assunto; Indexação de documentos; Indexação de imagens; Informação jurídica; Jurisprudência; Leitura técnica; Linguagem documental; Mediação da informação; Metacognição; Metodologia de indexação; Modelo de leitura documental; Modelo de leitura para indexação; Musicologia; Normalização; Obra musical; Partituras; Percurso gerativo de sentido; Polissemia; Princípios de análise e indexação; Processos cognitivos; Protocolo verbal; Recuperação da informação; Recuperação de imagens e filmes; Recuperação de informação; Superestrutura textual; Tesauro; Texto científico; Transferência de informações; Tratamento da informação; Tribunal de contas e Usuários.

Por meio da análise das fontes citadas, obtevese um conjunto total de 807 referências bibliográficas, o que significa uma média de 26 referências/citações por artigo. A análise das referências não contemplou aquelas que se caracterizavam como normas, pessoas jurídicas e instituições. As referências de autoria múltipla foram desdobradas para contemplar todos os autores do documento. Evidencia-se que 30 autores foram citados pelo menos em 5 diferentes artigos, representando a frente de pesquisa na temática.

$\mathrm{Na}$ Tabela IV são indicados os autores mais citados, com os respectivos dados. Neste conjunto de autores mais citados, Fujita aparece com 51 citações distribuídas em 21 trabalhos, seguida por Naves com 25 citações recebidas em 16 artigos; ambas pesquisadoras brasileiras.

\begin{tabular}{|c|c|c|}
\hline Autores & $\begin{array}{l}\text { N. de } \\
\text { citações }\end{array}$ & $\begin{array}{l}\text { N. de artigos } \\
\text { citado }\end{array}$ \\
\hline Fujita, M. S. L. & 51 & 21 \\
\hline Naves, M. M. L. & 25 & 16 \\
\hline Lancaster, E. W. & 17 & 13 \\
\hline Hjorland, B. & 18 & 11 \\
\hline Kobashi, N. Y. & 14 & 11 \\
\hline Dias, E. W. & 19 & 10 \\
\hline Pinto Molina, M. & 12 & 10 \\
\hline Guimarães, J. A. C. & 17 & 8 \\
\hline Albrechtsen, $\mathrm{H}$. & 10 & 8 \\
\hline Chaumier, J. & 9 & 8 \\
\hline Beghtol, C. & 8 & 8 \\
\hline Langridge, D. & 8 & 8 \\
\hline Neves, D. A. de B. & 10 & 7 \\
\hline Foskett, A. C. & 7 & 7 \\
\hline Rubi, M. P. & 11 & 6 \\
\hline Moreiro González, J. A. & 7 & 6 \\
\hline Cavalcanti, M. C. & 6 & 6 \\
\hline Chu, C. M. & 6 & 6 \\
\hline Cintra, A. M. C. & 6 & 6 \\
\hline Hutchins, J. & 6 & 6 \\
\hline O'brien, A. & 6 & 6 \\
\hline Pinto, M. C. F. & 6 & 6 \\
\hline Mai, J. E. & 9 & 5 \\
\hline Farrow, J. & 7 & 5 \\
\hline Tálamo, M. de F. G. M. & 7 & 5 \\
\hline Cesarino, M. A. N. & 6 & 5 \\
\hline Gil Leiva, I. & 5 & 5 \\
\hline Pinheiro, A. M. V. & 5 & 5 \\
\hline Šauperl, A. & 5 & 5 \\
\hline Todd, R. T. & 5 & 5 \\
\hline
\end{tabular}

Tabela IV. Autores mais citados

A partir destes autores citados, foi elaborada a rede de citação. Os círculos vermelhos representam os autores citados, enquanto que os círculos azuis representam os autores que foram citantes e citados ao mesmo tempo. Não houve autores que foram apenas citantes. Cabe esclarecer que quanto maior a espessura do seguimento, maior o número de vezes em que o autor foi citado. 


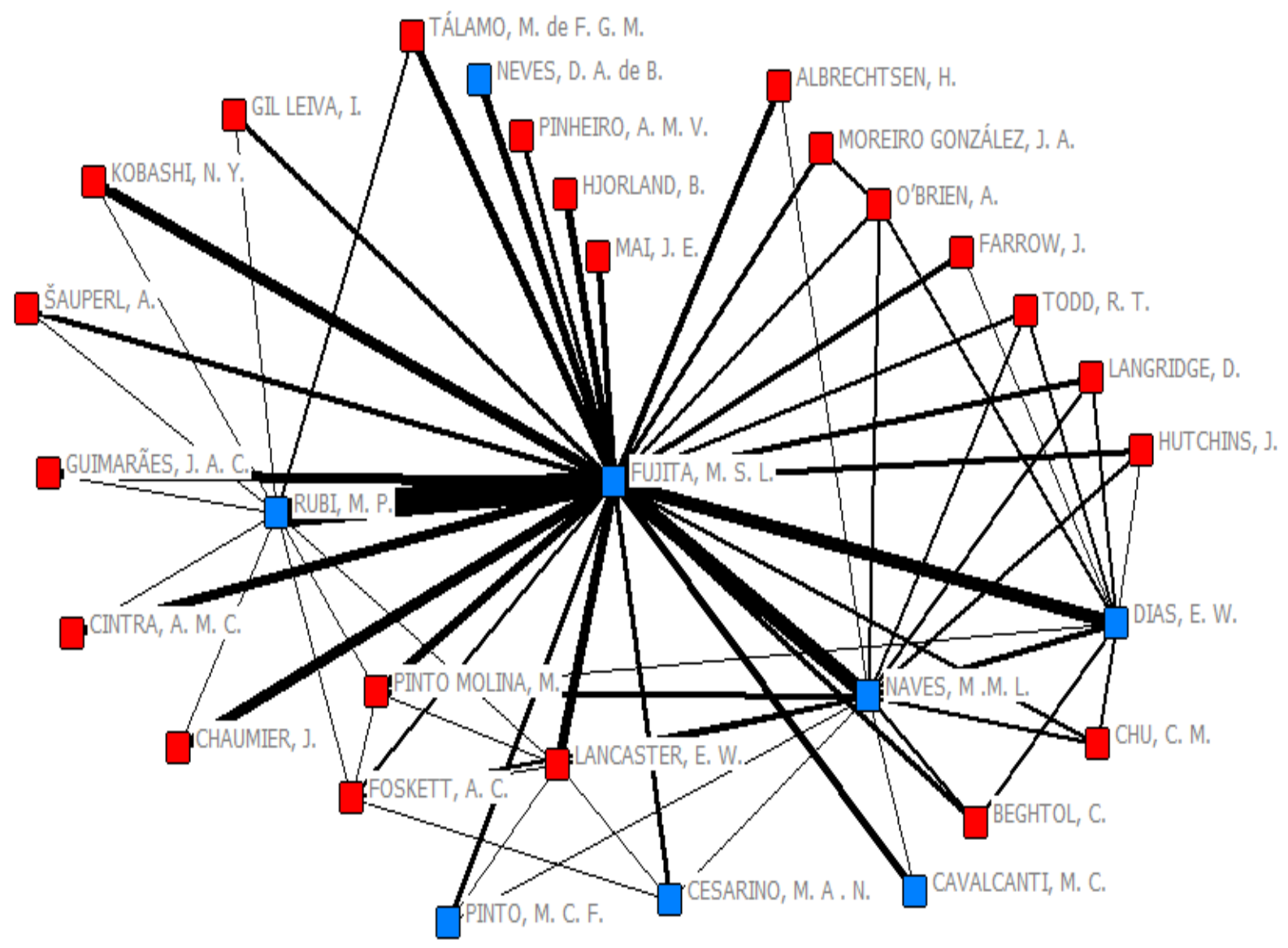

Figura II. Representação gráfica da rede de autores citantes e citados em análise de assunto

Na rede de autores citantes e citados, verifica-se que os autores Fujita, Naves, Dias, Rubi, Cavalcanti, Cesarino, Pinto e Neves formam o grupo de autores que citam e ao mesmo tempo são citados pelos demais autores da rede, podendo ser identificados como o núcleo de especialistas na temática análise de assunto. Dentre os autores brasileiros apenas citados estão: Tálamo, Pinheiro, Cintra, Guimarães e Kobashi. Os autores estrangeiros, maioria dentre os autores citados, são: Lancaster, Hjorland, Albrechtsen, Chaumier, Beghtol, Langridge, Foskett, Moreiro González, Chu, Hutchins, O’brien, Mai, Farrow, Gil Leiva, Sauperl e Todd. Em sua maioria, estes são autores clássicos da abordagem teórica da indexação no campo da Organização e Representação do Conhecimento.

Na sequência, são dispostos os principais resultados relacionados à categorização do corpus de pesquisa mediante análise interpretativa.

\subsection{Panorama das pesquisas no cenário nacional}

Dentre os estudos compilados nesta pesquisa, ganham destaque os de natureza conceitual. Ao consolidarem as concepções teóricas e metodológicas sobre análise de assunto hoje praticadas na área, tornam-se investigações de vasta contribuição para o enriquecimento da temática na Ciência da Informação brasileira.

O primeiro estudo refere-se ao de Cesarino e Pinto (1980), no qual é apresentada uma contextualização geral acerca da análise de assunto como operação fundamental para a recuperação da informação. Os diferentes níveis de análise de assunto são abordados, considerando a determinação do conteúdo informativo do documento; a compreensão das necessidades dos usuários; bem como a escolha ou elaboração de linguagens de indexação. Além de servir de base para outros estudos conduzidos no cenário nacional, apresenta uma das primeiras reflexões acerca da automação para a análise de textos, até então tarefa exclusiva dos indexadores.

Em Naves (1996) são abordadas as várias concepções e abordagens empregadas ao termo, assim como ao conceito de atinência. De modo elucidativo, a discussão estabelecida no estudo trabalha a abordagem conceitual da análise de assunto como etapa do processo de indexação. 
Considerada por muitos dos teóricos da área uma atividade intelectual e subjetiva, a imprecisão terminológica da análise de assunto ganha destaque no trabalho da autora, a qual chama a atenção para a influência negativa desta divergência conceitual na determinação de assunto dos documentos por parte dos indexadores.

Tomando como base as dificuldades inerentes à análise de assunto, Fujita (2003) discorre, mediante revisão de literatura, sobre os aspectos que envolvem a identificação de conceitos realizada durante a leitura documental na indexação. Observa que as concepções de análise de assunto orientadas para o conteúdo e para a demanda devem ser privilegiadas pelo indexador para garantir bons resultados na recuperação.

A prática da indexação foi objeto de estudo no trabalho apresentado por Silva e Fujita (2004). Neste, as autoras realizam uma ampla e consistente análise da evolução das tendências teóricas e metodológicas desenvolvidas pela literatura especializada até então. Por conseguinte, o estudo contribui para o entendimento da prática do indexador, com destaque especial para as especificidades do processo e suas etapas inerentes.

Por sua vez, Duarte (2007) estuda os fatores intervenientes no processo de indexação, com ênfase para a etapa da análise de assunto. Ao sistematizar as principais contribuições teóricas acerca da identificação de conceitos, da tematicidade e das concepções de análise de assunto, acentua a importância de se conduzir o processo tendo o usuário como ponto central. Em Neves, Dias e Pinheiro (2006), a intenção foi identificar semelhanças e divergências no uso das principais estratégias metacognitivas entre leitores proficientes indexadores e não-indexadores. $\mathrm{O}$ estudo revelou a necessidade de se introduzir o ensino de estratégias metacognitivas no treinamento de leitura do indexador.

Ao discorrerem sobre os aspectos teóricos e normativos da análise de assunto no processo de indexação, o estudo de Sousa e Fujita (2014) oferece à área uma importante contribuição ao apontar lacunas na utilização dos instrumentos que dão subsídios à análise de assunto, com destaque para a norma brasileira NBR $12676 / 1992$ que versa sobre os métodos para análise de documentos e a necessidade de atualização deste repertório.

Com proposta semelhante à realizada aqui, Lima e Maculan (2014) verificaram a literatura existente sobre a análise de assunto, tendo como corpus de análise os capítulos de revisões de literatura do Annual Review of Information Science and Technology (ARIST), dos anos de
1977, 1982, 1986 e 1989. Como conclusão, destacam a pouca atenção dada pela área para as questões que cercam a análise de assunto, muito em razão dos procedimentos interpretativos complexos que cercam a atividade ou, ainda, pelo encaminhamento de pesquisas destinadas à automação dos procedimentos.

Em Novellino (1996), o processo de representação da informação é objeto de estudo, com ênfase para a criação de instrumentos e elaboração de metodologias que viabilizem a transferência de Informações. O diferencial está na importância da interpretação do documento como parte de uma área de conhecimento, prerrogativa advinda com a análise de domínio.

Dias, Naves e Moura (2001) realizaram estudo do comportamento de busca de informação do usuário do sistema de informação, como forma de contribuir para o entendimento dos aspectos que envolvem o leitor e seus interesses e, assim, trazer novos entendimentos para as questões relativas à análise de assunto e com a prática do profissional indexador no momento da atribuição de conceitos. Nesta mesma direção, Dias (2004) analisou a percepção dos usuários quanto ao conteúdo dos documentos, tendo como pano de fundo a análise de assunto e o aperfeiçoamento do processo.

Rodrigues e Cervantes (2015) discorrem sobre as semelhanças entre os mapas conceituais e a atividade da análise de assunto, especificamente no que tange às etapas de extração e tradução de conceitos. Para avançar na questão, as autoras realizaram estudo teórico-metodológico para a aplicação dos mapas conceituais no processo de análise, síntese e representação da informação, com especial destaque no auxílio deste instrumento nas estratégias de cognição dos profissionais no tratamento temático da informação (Rodrigues e Cervantes, 2016).

Os temas de estudo com perspectiva mais específica são: observação dos elementos para análise e representação temática de partituras musicais, propondo uma análise conceitual a partir da leitura documental amparada por elementos básicos no âmbito da Musicologia (Cavalcanti e Carvalho, 2011); análise das classificações do conhecimento com base na análise de assunto e outras abordagens teóricas, buscando verificar os fatores intervenientes nos processos de organização e representação da informação no universo da ciência e tecnologia (Souza, 2012); reflexão acerca dos aspectos básicos para a análise de imagens e filmes a serem considerados na determinação de princípios e diretrizes para a elaboração de política de indexação e na recuperação em diversos ambientes informacionais 
(Cordeiro, 2013); uso de técnicas de análise de assunto e de conteúdo para a observação da construção imagética e discursiva do ciclo junino, tendo as letras de músicas como recurso informacional (Pinho; Marinho e Nascimento, 2014); investigação das estratégias metacognitivas para análise de assunto em literatura infanto-juvenil (Alves et. al, 2016); e reflexão acerca da contribuição teórica das ciências cognitivas para o entendimento dos processos ligados à mente (cognição humana e suas implicações), com atenção especial para a indexação de imagens e a análise conceitual desses documentos (Gonçalves; Oliveira e Neves, 2016).

A natureza aplicada da análise de assunto é fortemente trabalhada pela literatura a partir do desenvolvimento de metodologias que buscam contribuir para a leitura documental dos diversos tipos de documentos, com fins de identificação e seleção de conceitos representativos. Uma discussão prática sobre a sistematização dos procedimentos foi objeto de estudo de Fujita e Rubi (2006) ao discorrerem sobre os princípios de elaboração e uso de um modelo de leitura documental para a indexação de artigos científicos empregado na formação de indexadores em leitura documental. Essa proposição metodológica alia estratégias de exploração da estrutura textual e a identificação de conceitos de textos científicos por meio de questionamento, o que favorece o ensino da indexação.

Neste viés investigativo, Ferreira e Maculan (2016) apresentam uma proposta de modelo de leitura técnica de acórdãos direcionado para o contexto dos tribunais de contas, como forma de estabelecer parâmetros que auxiliem a prática da indexação de documentos jurisprudenciais. Temse, ainda, uma proposta de modelo de leitura técnica para estruturar a identificação dos conceitos representativos do conteúdo de documentos acadêmicos do tipo teses e dissertações (Maculan e Lima, 2011).

Considerando-se a leitura documental como um ato social, Fujita (2004) investigou as variáveis texto, leitor e contexto, o que viabilizou uma maior compreensão das dificuldades inerentes à análise de assunto. $\mathrm{O}$ estudo discorreu sobre as principais dificuldades no processo de leitura advindas com a combinação das variáveis, observadas a partir de revisão teórica, entrevistas com indexadores e diagnóstico de sistemas de informação brasileiros. A relevância desta investigação está, sobretudo, com a indicação de novas orientações para a formação do indexador em leitura documental. Em outro estudo, a referida autora observou a tarefa de catalogação de assuntos de livros em bibliotecas universitárias para desenvolver e apresentar o modelo de leitura documental para a indexação na catalogação de assuntos de livros em bibliotecas, metodologia adaptada do modelo de leitura documental para indexação de textos científicos, apresentado em estudos anteriores. Para tanto, a identificação de conceitos foi combinada com a exploração da estrutura textual de livros no processo de catalogação de assunto (Fujita, 2013). Ainda no contexto da leitura documental, Redigolo e Fujita (2015) debruçaram-se sobre a influência da ação mediadora inerente ao profissional da informação na representação de assunto dos documentos, sendo requerido uma ação consciente na condução do processo e atribuição de conceitos.

Objetivando retratar os diversos fatores que interferem no processo da análise de assunto, a principal contribuição do estudo de Naves (2001) advém com a indicação da necessidade de abordagens interdisciplinares para amenizar e tratar questões inerentes ao processo, principalmente da Linguística, Psicologia Cognitiva e Lógica. Considerando a relevância do estudo semiótico para a atividade de indexação, Redigolo e Almeida (2012) propuseram uma reflexão teórica acerca das relações e contribuições da Filosofia e da Semiótica no tocante às questões que envolvem a análise de assunto, bem como os conceitos de hábito, experiência, representação e tipos de signos gerados nesse processo.

Em Fujita, Boccato e Rubi (2010) os procedimentos, dificuldades e percepções de bibliotecários, usuários e gestores acerca do processo de indexação na catalogação em bibliotecas universitárias foram observadas sob a perspectiva da abordagem sociocognitiva. A ausência de procedimentos para análise e representação do livro, a incompatibilidade da linguagem de indexação e problemas relacionados à recuperação de assuntos nos catálogos, fruto da tendência de atuarem como bases de dados, foram alguns dos resultados identificados.

O estudo apresentado por Dal'Evedove e Fujita (2012) centrou esforços para compreensão do conhecimento profissional acerca da sistematicidade da catalogação de assunto em perspectiva sociocognitiva. A análise do processo em contexto de bibliotecas universitárias pela perspectiva profissional culminou na constatação de que muitas das ações desempenhadas pelos catalogadores de assunto em sua prática cotidiana são alicerçadas em experiências pessoais e profissionais; resultado que reforça a importância que manuais, procedimentos e instrumentos ocupam na prática profissional com fins de viabilizarem a padronização do processo. 
Tendo como ponto central a importância do aprimoramento e atualização de normas e procedimentos de análise de assunto, Redigolo (2014) observou a catalogação de assunto praticada em bibliotecas universitárias do Brasil e da Espanha, concluindo que a ausência de instrumentos metodológicos faz com que o profissional desenvolva seus próprios métodos e não se paute nas concepções trabalhadas e propostas pela literatura especializada, o que acarreta prejuízos na representação de assunto e recuperação da informação.

Apesar do avanço das pesquisas brasileiras acerca da indexação, observa-se que o primeiro estágio do processo, a análise de assunto, apresenta questões que não foram devidamente exploradas, sendo necessário novos esclarecimentos para o avanço da temática no cenário nacional.

\section{Tendências de pesquisa na Ciência da Informação brasileira}

A categorização das pesquisas brasileiras sobre análise de assunto publicadas em periódicos científicos viabilizou a compreensão do estado atual das discussões na temática, assim como o levantamento das principais lacunas a serem trabalhadas pelos teóricos da Ciência da Informação no Brasil. Na maioria dos casos, as conclusões dos estudos reforçam a escassez de investigações no campo da representação temática da informação e alertam para a necessidade de aprofundamento teórico da etapa de análise de assunto.

Como contributo à questão, são apresentados, de modo sumarizado, alguns dos pontos que merecem destaque e atenção pelos especialistas, quais sejam:

- Maior atenção aos fatores intrínsecos e extrínsecos interferentes no processo de indexação, especificamente na etapa de análise de assunto, dado a sua natureza subjetiva;

- Proposta de estudos que verifiquem a aplicação prática da análise de assunto durante a indexação realizada em diferentes contextos informacionais; assim como a atualização de normas e políticas específicas para oferecer maior suporte à prática profissional;

- A complexidade da sistematização de seus procedimentos exige um olhar verticalizado para os aspectos histórico-culturais envolvidos no processo;

- Abordagens complementares acerca do processamento da informação durante a atividade de análise de assunto, por decorrência da dificuldade em se compreender como a mente humana realiza determinadas funções cognitivas;

- Maior familiaridade com as atividades cognitivas e metacognitivas do profissional para a criação de novos saberes e conhecimento teórico-aplicado;

- Observações complementares que privilegiem o ponto de vista dos usuários para a criação de arcabouços teóricos e metodológicos em análise de assunto; e

- A deficiência na formação e falta de especialização do profissional reforçam a condução de estudos específicos e aplicados aos diversos contextos de informação.

\section{Considerações finais}

Ao objetivar a mediação da informação socializada para o indivíduo, produzindo novos conhecimentos, o ciclo de operações documentais é considerado a base epistemológica da Ciência da Informação, composto por produtores/autores e consumidores/usuários de informação como substrato para a produção de novos conhecimentos. Neste cenário, destaca-se o importante papel da indexação nos sistemas de recuperação da informação.

Considerando-se a relevância de estudos destinados à compreensão do conhecimento produzido em indexação, esta investigação foi pensada e construída de modo a verificar o atual estado da arte sobre a análise de assunto no meio acadêmico-científico da Ciência da Informação brasileira.

Mediante um amplo mapeamento bibliográfico, observou-se a produção do conhecimento na temática divulgada como artigos científicos em periódicos nacionais da área. A sistematização, análise e interpretação dos dados viabilizaram a postulação de hipóteses e recomendações que servirão de ponto de partida para outras pesquisas.

De modo geral, destaca-se a necessidade de pesquisas complementares que busquem subsídios para as múltiplas questões que envolvem a análise de assunto do ponto de vista teórico e prático. Alguns desses caminhos a serem percorridos foram aqui evidenciados, tomando-se como base os resultados e considerações da atual literatura periódica brasileira na temática.

\section{Referências}

Chan, L. M (1994). Cataloging and classification: an introduction. 2nd ed. New York: McGraw-Hill, 1994. 
Chu, C. M., O'Brien, A (1993). Subject analysis: the critical first estage in indexing. // Journal of Information Science. 1:19.

Cleveland, D. B.; Cleveland, A. D (1990). Introduction to indexing and abstracts. 2nd ed. Englewood: Libraries Unlimited, 1990.

Cesarino, A. da N.; Pinto, M. C. M. F (1980). Análise de assunto. // R. Bibliotecon. Brasília 8:1.

Fujita, M. S. L. (2003). A identificação de conceitos no processo de análise de assunto para indexação. // Revista Digital de Biblioteconomia e Ciência da Informação. 1:1 6090.

Fujita, M. S. L.; Rubi, M. P.; Boccato, V. R. C (2009). As diferentes perspectivas teóricas e metodológicas sobre indexação e catalogação de assuntos. // Fujita, M. S. L. (Org.). A indexação de livros: a percepção de catalogadores e usuários de bibliotecas universitárias. São Paulo: Cultura Acadêmica, 2009. 19-42.

ISO 5963 Documentation. Methods for Examining Documents, Determining their Subjects, and Selecting Indexing Terms. Geneve: International Organization for Standardization, 1985.

Lancaster. F. W (2004). Indexação e resumos: teoria e prática. Tradução de: Antonio Agenor Briquet de Lemos. 2 ed. Brasília: Briquet de Lemos, 2004.
Langridge, D (1989). Subject analysis: principles and practice. London: Bowker-Sour, 1989.

Mai, J-E. (1997). The concept of subject: on problems in indexing. // Knowledge Organization for Information Retrieval. Proceedings of the 6th International Study Conference on Classification Research. 6, 60-67, 1997.

Mai, J-E. (2000). Deconstructing the Indexing Process. Advances in Librarianship 23: 269-298.

Neves, D. A. de B.; Dias, E. W.; Pinheiro, A. M. V (2006). Uso de estratégias metacognitivas na leitura do indexador. // Ci. Inf. 35:3 (Sep./Dec. 2006) 141-152.

Salvador, A. D (1986). Métodos e técnicas de pesquisa bibliográfica. // Porto Alegre: Sulina, 1986.

Tamayo, A. M. M.; Valdez, J. C. Indización y clasificación en bibliotecas. Buenos Aires: Alfagrama, 2008.

Tartarotti, R. C. D., Boccato, V. R. C (2013). A abordagem teórica bakhtiniana e o processo de indexação: diálogos. // Versão Beta: sob o signo da palavra. 11:74.

Van Slype, G (1991). Lenguages de indización: concepción, contrucción y utilización en los sistemas documentales. Trad. Pedro Hípola e Félix de Moya. Madrid: Fundación Germán Sánchez Ruipérez, 1991.

Enviado: 2017-04-01. Segunda versión: 2017-09-28. Aceptado: 2018-03-23.

Dal'Evedove, Paula Regina; Tartarotti, Roberta Cristina Dal'Evedove; Fujita, Mariângela Spotti Lopes. Estudos sobre análise de assunto no Brasil: estado da arte e perspectivas futuras. // Scire. 24:1 (en.jun. 2018) 35-44. ISSN 1135-3716. 